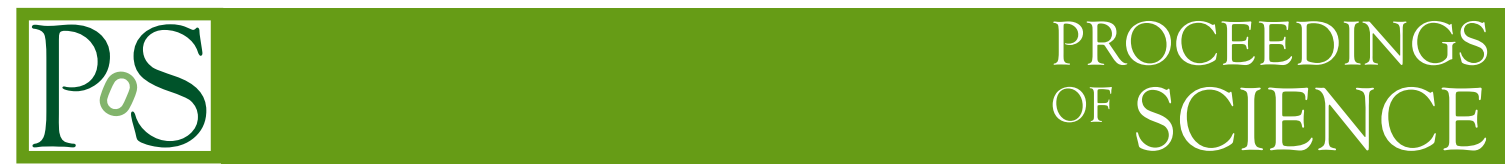

\title{
Five-loop massive tadpoles
}

\section{Thomas Luthe}

Institut für Theoretische Teilchenphysik, Karlsruhe Institute of Technology, Karlsruhe, Germany

E-mail: thomas.lutheakit.edu

\section{York Schröder*}

Grupo de Fisica de Altas Energias, Universidad del Bio-Bio, Casilla 447, Chillan, Chile

E-mail: yschroeder@ubiobio.cl

We provide an update on a long-term project that aims at evaluating massive vacuum integrals at the five-loop frontier, with high precision and in various space-time dimensions. A number of applications are sketched, mainly concerning the determination of anomalous dimensions, for quantum field theories in four, three and two dimensions.

Loops and Legs in Quantum Field Theory

24-29 April 2016

Leipzig, Germany

${ }^{*}$ Speaker. 


\section{Introduction}

In the light of ongoing large-scale experimental effort to search for new particles at the highenergy frontier, with the daunting task of filtering signals out of the overwhelming Standard Model (and, in particular, QCD) background processes, the importance of evaluating the fundamental building blocks that enter high-precision perturbative expansions cannot be overstated. One class of such fundamental building blocks are so-called master integrals, which arise in modern Feynmandiagrammatic multi-loop calculations. In order to optimize the theoretical effort that is invested in obtaining the necessary high-precision determinations of physical observables, it is often desired to once and for all tabulate universal elements (such as these master integrals) that are required often, but are difficult or time-consuming to obtain.

Master integrals come in all sorts of flavors, and can be classified according to number of loops, external invariants, propagator masses, space-time dimension, finiteness, or number content, to name a few properties. Integration being an art rather than a mechanic task such as differentiation, only a small subset of master integrals is expected to be amenable to analytic methods, while the majority will need to be approximated numerically, a task complicated by ultraviolet and infrared divergences and -subdivergences that can be present.

In this contribution, pushing on the loop-frontier, we will focus on one of the simplest possible integral classes: fully massive tadpoles, which correspond to vacuum diagrams without external lines, in which all particles/propagators share a common mass. The mass-dependence then follows trivially from dimensional arguments, such that, without loss of generality, we can set the mass parameter to unity, leaving us with a zero-scale problem of computing pure numbers. Indeed, over the years there have already been a fair number of talks concerning this very class of basic integrals at this conference series, elucidating any subset of aspects listed above. We wish to continue this discussion, adding news at five loops.

\section{Method}

As mentioned in the introduction, we focus on the specific class of fully massive tadpoles up to 5 loops. The 5-loop integral family needs 15 propagators, of which maximally 12 can be present in a vacuum graph, see figure 1 for examples. There exist a number of viable methods evaluating such integrals, such as integration in coordinate space, differential equations (in a mass ratio, letting $M / m \rightarrow 1$ in the end), or numerical solutions of difference equations (in a symbolic propagator power $X$ that is set to unity the end) via factorial series. We favor the latter approach, as formulated algorithmically by Laporta $[1,2]$.

Laporta's setup, based on integration by parts [3], has already been successfully applied to the evaluation of a large number of Feynman integrals, and in particular also to 4-loop massive tadpoles in $3 \mathrm{~d}[4]$ and $4 \mathrm{~d}[5,6]$. This makes a 5-loop extension natural; for some first results, see [7]. Our method has been developed and described in more detail in [8] and [9], where the latter reference in particular contains a substantial fine-tuning of the Laporta approach in order to delay intermediate expression swell, implementing ideas such as using coupled equations, reducing recurrence relations, and predicting instability factors, and presents the program TIDE. Regarding results given earlier [7] and below, we would like to note that, in order to obtain convention- 


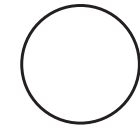

$\mathrm{J}$

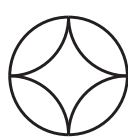

30876

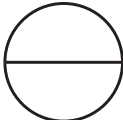

7

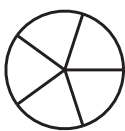

32596

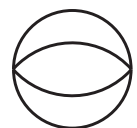

51

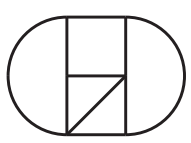

32279

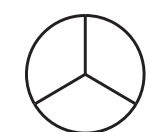

63

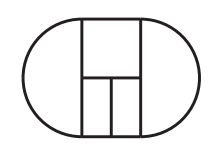

32745

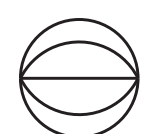

841

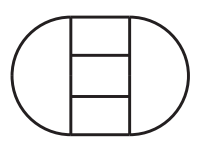

31740

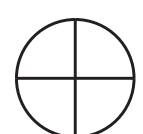

1011

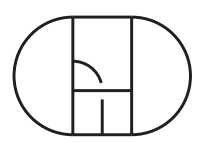

30699

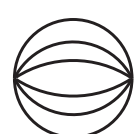

28686

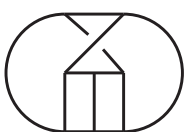

30527

Figure 1: Momentum integrals $I_{n}$ discussed here. Solid lines denote massive propagators $1 /\left(k^{2}+m^{2}\right)$.

independent results, we choose to divide each $L$-loop integral by the respective power of the 1loop massive tadpole $J^{L}$ which, picking a concrete momentum integral measure and working in Euclidean space-time, reads $J=\int \mathrm{d}^{d} k /\left(k^{2}+1\right)=\pi^{d / 2} \Gamma(1-d / 2)$.

\section{Results and checks}

We have obtained numerical results for the $\varepsilon$-expansions of the set of fully massive tadpoles at 5 loops, most of which are new. In practice, we aimed at about 300 digits of final precision for the first ten $\varepsilon$-orders in expansions around $d_{0}=2,3$ and 4 dimensions. Our results cover the full set of 63 5-loop sectors with 5 to 11 lines, amassing a total of 103 master integrals. A number of results for four-dimensional expansions of 5-loop masters have already been given elsewhere $[9,7]$. Presently, mainly due to limitations in computer resources, we are unable to obtain results for the four 5-loop sectors with 12 lines, depicted as $I_{32745}, I_{31740}, I_{30699}$ and $I_{30527}$ in figure 1, which together contain a total of 9 masters integrals. Further optimizations of the code TIDE [9] are under way, and we are confident that these last four sectors will be solved in the near future.

As has already been mentioned above, in the program TIDE, a number of new ideas and improvements over the original Laporta algorithm have been implemented. Therefore, it is important to perform cross-checks on the results the code delivers. To this end, we have performed successful checks against known lower-order results, in particular at four loops in $4 \mathrm{~d}[5,6]$ as well as $3 \mathrm{~d}$ [4].

Furthermore, there is an important internal check that is inherent in the method of difference equation that we are employing. Except for totally symmetric graphs (which correspond to our 5loop sector numbers 28686, 30876 and 31740, see figure 1), for all so-called corner integrals (those with all propagator powers equal to unity) TIDE generates a number of independent results coming from deriving difference equations with power $X$ on inequivalent lines. All of these results have to coincide at $X=1$, and they do within our numerical precision for expansions around different values (we have explicitly checked $4 \mathrm{~d}, 3 \mathrm{~d}$, and $2 \mathrm{~d}$ expansions) of the space-time dimension.

We can also look at specific sets of integrals that are known analytically. As an example, we take the 2-loop massive sunset vacuum integral, for which a $d$-dimensional analytic solution in terms of hypergeometric functions is available $[10,6]$. The normalized 2-loop sunset integral can then be expanded to all orders in $\varepsilon$ in various dimensions, for example:

$$
\begin{aligned}
& I_{7} / J^{2} \stackrel{4-2 \varepsilon}{=}-\frac{3}{2}-\frac{3}{2} \varepsilon+3\left(3 H_{2}-1\right) \varepsilon^{2}+3\left(3 H_{2}-6 H_{3}-2\right) \varepsilon^{3}+6\left(3 H_{2}-3 H_{3}+6 H_{4}-2\right) \varepsilon^{4}+\ldots, \\
& I_{7} / J^{2} \stackrel{2-2 \varepsilon}{=} 6 H_{2} \varepsilon^{2}-12 H_{3} \varepsilon^{3}+24 H_{4} \varepsilon^{4}-48 H_{5} \varepsilon^{5}+96 H_{6} \varepsilon^{6}+\ldots,
\end{aligned}
$$


where the rather compact expressions are due to having introduced the set of transcendentals

$$
H_{n}=h_{n}+h_{1} \hat{C}_{n-1}\left(1-\frac{3^{\varepsilon / 2} \Gamma(1-\varepsilon)}{\Gamma^{2}(1-\varepsilon / 2)}\right), \quad h_{n}={ }_{n+1} F_{n}\left(\frac{1}{2}, \ldots, \frac{1}{2} ; \frac{3}{2}, \ldots, \frac{3}{2} ; \frac{3}{4}\right),
$$

where the operator $\hat{C}_{n}$ picks out the coefficient of order $\varepsilon^{n}$. For example, $H_{1}=2 \pi / \sqrt{27}$, while $3 H_{2}=\sqrt{3} \mathrm{Cl}_{2}(2 \pi / 3)$ is related to the Clausen function $\mathrm{Cl}_{n}(x)=\sum_{k>0} \sin (k x) / k^{n}=\operatorname{ImLi}_{\mathrm{n}}\left(\mathrm{e}^{\mathrm{ix}}\right)$ that had been studied in relation with massive 3-loop vacuum integrals [11]. We observe that the corresponding numerical results for $I_{7}$ delivered by TIDE agree perfectly with the expansions given above, in four as well as two dimensions. Generalizing the sunset integral to $(L+1)$ lines connecting two vertices, the corresponding integral class $S_{L}$ can in principle be studied numerically in any dimension and at any loop order using coordinate-space techniques [12]. Our lower-loop results $I_{7}, I_{51}$ and $I_{841}$ compare favorably with $S_{2}, S_{3}$ and $S_{4}$, respectively. At five loops, the corresponding result for $S_{5} / J^{5}$ is easily superseeded in precision by TIDE, which delivers at least 250 digits of (we only show 50 digits here; recall that we divide by the 1-loop tadpole, which corresponds to multiplying $4 \mathrm{~d}$ 5-loop integrals by $\varepsilon^{5}$ )

$$
\begin{aligned}
I_{28686} / J^{5}= & -2.9999999999999999999999999999999999999999999999999 \varepsilon^{0} \\
& -1.5000000000000000000000000000000000000000000000000 \varepsilon^{1} \\
& +0.5416666666666666666666666666666666666666666666666 \varepsilon^{2} \\
& -0.8798611111111111111111111111111111111111111111111 \varepsilon^{3} \\
& -1.2132523148148148148148148148148148148148148148148 \varepsilon^{4} \\
& +135.95072868792871461956492733702218574897992953584 \varepsilon^{5} .
\end{aligned}
$$

Similarly, for another check at five loops, we consider the class $W_{L}$ of wheel-type integrals with $L$ spokes. In four dimensions, their leading $\varepsilon$-order is known to all loop orders [13]

$$
\frac{W_{L}}{J^{L}}=\frac{(-1)^{L} \Gamma(2 L-1)}{\Gamma(L) \Gamma(L+1)} \zeta(2 L-3) \varepsilon^{L-1}+\mathscr{O}\left(\varepsilon^{L}\right),
$$

with the leading term of our result for

$$
\begin{aligned}
I_{32596} / J^{5}= & -14.116889883346919575757165697897154634398089847913 \varepsilon^{4} \\
& +235.07729596783467131454388080950411779239347239580 \varepsilon^{5} \\
& -2267.7386832930084122962994480580205855487545413738 \varepsilon^{6}
\end{aligned}
$$

matching the five-loop case $W_{5} / J^{5}=-14 \zeta(7) \varepsilon^{4}$ to 250 digits, and our lower-loop results $I_{1011}$ and $I_{63}$ matching $W_{4}$ and $W_{3}$ to tens of thousands digits. In the same spirit, considering the family $Z_{L}$ of $L$-loop zigzag-type integrals, we can compare with known all-loop-order results in four dimensions, which in terms of the previous class reads $[14,15,16]$

$$
\frac{Z_{L}}{J^{L}}=\frac{W_{L}}{J^{L}} \frac{4}{L}\left(1+\frac{(-1)^{L}-1}{2^{2 L-3}}\right)+\mathscr{O}\left(\varepsilon^{L}\right) .
$$

The leading term of our result for

$$
\begin{aligned}
I_{32279} / J^{5}= & -11.117050783135699165908767987094009274588495755231 \varepsilon^{4} \\
& +181.78223928612340820790788236018642961198741994209 \varepsilon^{5} \\
& -1725.9996137403520805951673992442117288607704957542 \varepsilon^{6}
\end{aligned}
$$


agrees with $Z_{5} / J^{5}=-\frac{441}{40} \zeta(7) \varepsilon^{4}$ predicted by eq. (3.6) to our 5-loop numerical precision of 250 digits, while the lower-loop ones agree to much higher precision (at 4 and 3 loops, the two integral families actually coincide, $W_{4}=Z_{4}$ and $W_{3}=Z_{3}$ ).

Another standard method to evaluate Feynman integrals is integration over the Feynmanparametric representation (see, e.g. [17]), where the integrand is given by two characteristic polynomials (Symanzik polynomials, usually called $U$ and $F$ ) that are fixed by the topology of the underlying graph. In most practical cases, this amounts to numerical integration, and a number of public implementations exist to facilitate this task $[18,19,20]$. We can use this method here for some low-precision checks of our results, and to estimate the magnitude of the missing integrals. It turns out that for the class of fully massive integrals without external legs, the graph polynomials coincide in general (since $F=\left(x_{1}+\cdots+x_{N}\right) U=U$ ). Therefore, a fully massive $L$-loop vacuum integral in $d$ dimensions, having $N$ propagators with powers $a_{1}, \ldots, a_{N}$, normalized to $J^{L}$ (where $J$ is the 1-loop massive tadpole, containing the momentum integral measure of choice) can be represented as an integral over the $N$-dimensional simplex with a particularly simple integrand,

$$
\frac{I_{\vec{a}}}{J^{L}}=\frac{\Gamma(A-L d / 2)}{[\Gamma(1-d / 2)]^{L}} \int_{0}^{\infty} \mathrm{d}^{N} x \delta(1-X) \frac{p_{\vec{a}}(\vec{x})}{[U(\vec{x})]^{d / 2}} .
$$

We have denoted the sum of indices as $A=a_{1}+\cdots+a_{N}$, and $X=x_{1}+\cdots+x_{N}$ is the sum of integration variables. The integrand contains a numerator $p_{\vec{a}}(\vec{x})=\prod_{i=1}^{N} x_{i}^{a_{i}-1} / \Gamma\left(a_{i}\right)$ that is different from unity for integrals with dots, and the graph polynomial $U$ that is homogeneous of degree $L$. Typically, a so-called primary sector decomposition [21, 22] is performed on eq. (3.8), whence the integration domain simplifies to a $(N-1)$-dimensional hypercube

$$
\frac{I_{\vec{a}}}{J^{L}}=\frac{\Gamma(A-L d / 2)}{[\Gamma(1-d / 2)]^{L}} \int_{0}^{1} \frac{\mathrm{d}^{N-1} z}{(1+Z)^{A-L d / 2}} \sum_{s=1}^{N} \frac{p_{\vec{a}}\left(\vec{z}_{s}\right)}{\left[U\left(\vec{z}_{s}\right)\right]^{d / 2}} .
$$

Here, $Z=z_{1}+\cdots+z_{N-1}$ is the sum of integration variables, and $\vec{z}_{s}=\left(z_{1}, \ldots, z_{s-1}, 1, z_{s}, \ldots, z_{N-1}\right)$ fits the $(N-1)$ integration variables into an $N$-vector. All polynomials in eq. (3.9) are positive semidefinite, such that we can use a simple numerical integration routine to verify many of our results to low precision, and confirm that the 12-line master integrals are indeed finite in $d=4$.

\section{Applications}

As a first application of our (4d) five-loop master integrals, we have set out to determine the set of anomalous dimensions in QCD [23]. The calculation follows standard procedures, utilizing Qgraf [24] for diagram generation and FORM [25] for most computer algebraic manipulations. To isolate the overall ultraviolet divergences of the (massless) two- and three-point functions, one can either re-route external momenta through the diagram in clever ways in order to systematically cancel infrared divergences [26, 27], or introduce masses into all propagators, at the expense of one additional (mass-) counterterm [28, 29]. We choose to follow the second option, and map all integrals onto fully massive tadpoles by recursively applying the identity [30, 31]

$$
\frac{1}{(k+q)^{2}}=\frac{1}{k^{2}+m^{2}}\left(1+\frac{m^{2}-2 k q-q^{2}}{(k+q)^{2}}\right)
$$


where $k(q)$ are loop (external) momenta and where integrals that are finite by naive power-counting are dropped, eventually ending the recursion. Reduction of the large number of resulting scalar integrals to master integrals is done via integration by parts [3], for which a number of public codes are available $[32,33,34]$. However, due to the complexity at five loops, we found it necessary to use our own in-house programs crusher [35] and TIDE [9] for the integral reduction. The latter is written almost entirely in $\mathrm{C}++$, expect that it uses Fermat [36] for the polynomial algebra, and runs all time-critical code in parallel. Along these lines, we have been able to partially generalize results for the 5-loop SU(3) QCD Beta function presented by the Karlsruhe group [37] to a general gauge group. Concretely, so far we have completely reproduced the Beta function up to four loops, and obtained the $N_{\mathrm{f}}^{4}$ and $N_{\mathrm{f}}^{3}$ contributions at five loops for a general gauge group [23], confirming the corresponding $S U(3)$ results.

The ability to change the space-time dimensionality in our setup and demand $\varepsilon$-expansions around any desired integer dimension opens up a number of other fields of application (besides the obvious, evaluating the remaining renormalization constants of QCD). In particular, there are interesting questions related to renormalization-group functions in three-dimensional field theories, such as whether an analogue of Zamolodchikov's two-dimensional $c$-theorem can be proven in odd dimensional supersymmetric theories [38]. Furthermore, allowing for zero-mass propagators as well, even the constant parts of $3 \mathrm{~d}$ expansions of zero-scale master integrals do contribute to physical observables, such as for example in QCD thermodynamics, where they enter an effective theory treatment of the strongly interacting gluons present in the hot early-universe plasma, see e.g. [39]. Going finally to two dimensions, massive tadpoles can be used to study non-trivial properties of QCD-like theories such as the Gross-Neveu (GN) model, where evanescent operators spoil multiplicative renormalizability [40]. The GN model is particularly interesting due to its connection with problems in condensed-matter theory, such as the evaluation of critical exponents.

\section{Conclusions}

In the era of highly automated perturbative calculations, one key building block are master integrals. We have reported progress on evaluating the set of five-loop massive vacuum integrals, which play an important role in determining anomalous dimensions of various quantum field theories. Since we are able to obtain high-precision numerical results for the $\varepsilon$-expansions around any number of dimensions $d=d_{0}-2 \varepsilon$, applications include determinations of (a) the QCD Beta function [23] and other anomalous dimensions at $d_{0}=4$; (b) effective theory contributions to thermodynamic observables [39], or renormalization functions of supersymmetric gauge theories [38] at $d_{0}=3$; (c) Beta functions of QCD-like models relevant for critical exponents in condensed matter systems such as graphene [40] at $d_{0}=2$.

As an open challenge, due to the complexity of the corresponding difference- and recursion relations, the evaluation of the missing 12-line integrals is left for future work. These four sectors of trivalent 5-loop graphs are finite (at $d_{0}=4$ ), however, such that they are not expected to contribute to the anomalous dimensions. 


\section{Acknowledgments}

Y.S. acknowledges support from FONDECYT grant 1151281 and UBB project GI-152609/VC. All diagrams were drawn with Axodraw [41].

\section{References}

[1] S. Laporta, Calculation of master integrals by difference equations, Phys. Lett. B 504 (2001) 188 [hep-ph/0102032].

[2] S. Laporta, High precision calculation of multiloop Feynman integrals by difference equations, Int. J. Mod. Phys. A 15 (2000) 5087 [hep-ph/0102033].

[3] K. G. Chetyrkin and F. V. Tkachov, Integration by Parts: The Algorithm to Calculate beta Functions in 4 Loops, Nucl. Phys. B 192 (1981) 159;

[4] Y. Schröder and A. Vuorinen, High precision evaluation of four loop vacuum bubbles in three-dimensions, hep-ph/0311323.

[5] S. Laporta, High precision epsilon expansions of massive four loop vacuum bubbles, Phys. Lett. B 549 (2002) 115 [hep-ph/0210336].

[6] Y. Schröder and A. Vuorinen, High-precision epsilon expansions of single-mass-scale four-loop vacuum bubbles, JHEP 0506 (2005) 051 [hep-ph/0503209].

[7] T. Luthe and Y. Schröder, Fun with higher-loop Feynman diagrams, arXiv:1604.01262.

[8] J. Möller, Fully massive tadpoles at 5-loop: reduction and difference equations, $\mathrm{PhD}$ thesis, Bielefeld University 2012.

[9] T. Luthe, Fully massive vacuum integrals at 5 loops, $\mathrm{PhD}$ thesis, Bielefeld University 2015.

[10] A. I. Davydychev and J. B. Tausk, Two loop selfenergy diagrams with different masses and the momentum expansion, Nucl. Phys. B 397 (1993) 123.

[11] D. J. Broadhurst, Massive three - loop Feynman diagrams reducible to SC* primitives of algebras of the sixth root of unity, Eur. Phys. J. C 8 (1999) 311 [hep-th/9803091].

[12] S. Groote, J. G. Körner and A. A. Pivovarov, On the evaluation of a certain class of Feynman diagrams in x-space: Sunrise-type topologies at any loop order, Annals Phys. 322 (2007) 2374 [hep-ph/0506286].

[13] D. J. Broadhurst, Evaluation of a Class of Feynman Diagrams for All Numbers of Loops and Dimensions, Phys. Lett. B 164 (1985) 356.

[14] D. I. Kazakov, The Method Of Uniqueness, A New Powerful Technique For Multiloop Calculations, Phys. Lett. B 133 (1983) 406.

[15] D. J. Broadhurst and D. Kreimer, Knots and numbers in Phi**4 theory to 7 loops and beyond, Int. J. Mod. Phys. C 6 (1995) 519 [hep-ph/9504352].

[16] F. Brown and O. Schnetz, Proof of the zig-zag conjecture, arXiv:1208.1890.

[17] V. A. Smirnov, Analytic tools for Feynman integrals, Springer Tracts Mod. Phys. 250 (2012) 1.

[18] C. Bogner and S. Weinzierl, Resolution of singularities for multi-loop integrals, Comput. Phys. Commun. 178 (2008) 596 [arXiv:0709.4092]. 
[19] S. Borowka, G. Heinrich, S. P. Jones, M. Kerner, J. Schlenk and T. Zirke, SecDec-3.0: multi-scale integrals beyond one loop, Comput. Phys. Commun. 196 (2015) 470 [arXiv:1502.06595].

[20] A. V. Smirnov, FIESTA4: Optimized Feynman integral calculations with GPU support, Comput. Phys. Commun. 204 (2016) 189 doi:10.1016/j.cpc.2016.03.013 [arXiv:1511.03614].

[21] M. Roth and A. Denner, High-energy approximation of one loop Feynman integrals, Nucl. Phys. B 479 (1996) 495 [hep-ph/9605420].

[22] T. Binoth and G. Heinrich, Numerical evaluation of multiloop integrals by sector decomposition, Nucl. Phys. B 680 (2004) 375 [hep-ph/0305234].

[23] T. Luthe, A. Maier, P. Marquard and Y. Schröder, Towards the five-loop Beta function for a general gauge group, JHEP 1607 (2016) 127 [arXiv:1606.08662].

[24] P. Nogueira, Automatic Feynman graph generation, J. Comput. Phys. 105 (1993) 279;

[25] J. Kuipers, T. Ueda, J. A. M. Vermaseren and J. Vollinga, FORM version 4.0, Comput. Phys. Commun. 184 (2013) 1453 [arXiv:1203.6543].

[26] A. A. Vladimirov, Method for Computing Renormalization Group Functions in Dimensional Renormalization Scheme, Theor. Math. Phys. 43 (1980) 417.

[27] K. G. Chetyrkin and V. A. Smirnov, $R^{*}$ Operation Corrected, Phys. Lett. B 144 (1984) 419.

[28] T. van Ritbergen, J. A. M. Vermaseren and S. A. Larin, The Four loop beta function in quantum chromodynamics, Phys. Lett. B 400 (1997) 379 [hep-ph/9701390].

[29] M. Czakon, The Four-loop QCD beta-function and anomalous dimensions, Nucl. Phys. B 710 (2005) 485 [hep-ph/0411261].

[30] M. Misiak and M. Münz, Two loop mixing of dimension five flavor changing operators, Phys. Lett. B 344 (1995) 308 [hep-ph/9409454].

[31] K. G. Chetyrkin, M. Misiak and M. Münz, Beta functions and anomalous dimensions up to three loops, Nucl. Phys. B 518 (1998) 473 [hep-ph/9711266].

[32] A. von Manteuffel and C. Studerus, Reduze 2 - Distributed Feynman Integral Reduction, arXiv: 1201.4330.

[33] R. N. Lee, LiteRed 1.4: a powerful tool for reduction of multiloop integrals, J. Phys. Conf. Ser. 523 (2014) 012059 [arXiv:1310.1145].

[34] A. V. Smirnov and V. A. Smirnov, FIRE4, LiteRed and accompanying tools to solve integration by parts relations, Comput. Phys. Commun. 184 (2013) 2820 [arXiv:1302.5885].

[35] P. Marquard and D. Seidel, crusher (unpublished).

[36] R. H. Lewis, fermat, http://home.bway.net/lewis /

[37] P. A. Baikov, K. G. Chetyrkin and J. H. Kühn, Five-Loop Running of the QCD coupling constant, arXiv: 1606.08659 .

[38] J.A. Gracey, I. Jack, C. Poole and Y. Schröder, The a-function for $N=2$ supersymmetric gauge theories in three dimensions, arXiv:1609.06458.

[39] K. Kajantie, M. Laine, K. Rummukainen and Y. Schröder, Four loop vacuum energy density of the SU $(N(c))+$ adjoint Higgs theory, JHEP 0304 (2003) 036 [hep-ph/0304048].

[40] J. A. Gracey, T. Luthe and Y. Schröder, Four loop renormalization of the Gross-Neveu model, arXiv:1609.05071.

[41] J. C. Collins and J. A. M. Vermaseren, Axodraw Version 2, arXiv:1606.01177. 